\title{
Functional connectivity in relation to motor performance and recovery after stroke
}

\section{Kelly P. Westlake and Srikantan S. Nagarajan*}

Biomagnetic Imaging Laboratory, Department of Radiology and Biomedical Imaging, University of California San Francisco, CA, USA

\section{Edited by:}

Barry Horwitz, National Institutes of Health, USA

\section{Reviewed by:}

Christian Gerloff, University Medical Center Hamburg-Eppendorf, Germany

Cecile Gallea, Hôpital de la Pitié

Salpétrière, France

${ }^{*}$ Correspondence:

Srikantan S. Nagarajan, Biomagnetic Imaging Laboratory, Department of Radiology and Biomedical Imaging, University of California San Francisco, 513 Parnassus Avenue Suite S362, Box 0628, San Francisco, CA 94143, USA. e-mail: sri@radiology.ucsf.edu
Plasticity after stroke has traditionally been studied by observing changes only in the spatial distribution and laterality of focal brain activation during affected limb movement. However, neural reorganization is multifaceted and our understanding may be enhanced by examining dynamics of activity within large-scale networks involved in sensorimotor control of the limbs. Here, we review functional connectivity as a promising means of assessing the consequences of a stroke lesion on the transfer of activity within large-scale neural networks. We first provide a brief overview of techniques used to assess functional connectivity in subjects with stroke. Next, we review task-related and resting-state functional connectivity studies that demonstrate a lesion-induced disruption of neural networks, the relationship of the extent of this disruption with motor performance, and the potential for network reorganization in the presence of a stroke lesion. We conclude with suggestions for future research and theories that may enhance the interpretation of changing functional connectivity. Overall findings suggest that a network level assessment provides a useful framework to examine brain reorganization and to potentially better predict behavioral outcomes following stroke.

Keywords: stroke, functional connectivity, brain, recovery of function, neuroimaging, neuroplasticity

\section{INTRODUCTION}

Stroke lesions cause neural dysfunction both at the lesion site and in remote brain regions. Historically, reduced neural function of distant, structurally intact regions was thought to be due to edema and increased pressure on the remaining neurons. It was not until 1914 when Constantin von Monakow coined the term diaschisis that disruption in the transfer of information between connected brain regions became more widely acknowledged. He described diaschisis associative - cortical dysfunction due to lesions of connected areas within the ipsilesional hemisphere, and diaschisis commissuralis - cortical dysfunction due to lesions of the interconnections to the contralesional hemisphere (Von Monakow, 1914). Years later, Geschwind (1965a,b), echoing the ideas of Wernicke (1874), provided further theoretical support for non-local effects of brain lesions with a thorough account of "disconnection syndromes." He contended that the various forms of aphasia, apraxia, and agnosia were the result of anatomical disconnections caused by white matter lesions or lesions of association cortices (i.e., posterior sensory areas). Nevertheless, with a lack of empirical evidence supporting these principles, the prevailing belief for many years was that a reasonable symptomatic explanation could be obtained merely by defining the locus of a lesion, which inversely confirmed the function of that region. Even with the advent of functional neuroimaging, regional shifts of activity have come to define plasticity following stroke and yet still do not sufficiently capture the widely variable recovery of motor function. Therefore, in order to comprehend and treat persistent motor impairments, it appears crucial to move beyond segregated perspectives of brain function and characterize the lesioned brain as an integrated and reorganized functional network.
Recently, the creation of anatomically connected network maps in healthy brains has led to a conceptualization of the impact of lesions in brains affected by stroke. To a certain extent, these models have shown that the unpredictability in functional outcomes may indeed be due to the lesion location (Alstott et al., 2009). However, the reasons extend beyond the localized function of the region and emphasize the importance of whether the lesion occurred at a node of a large-scale brain network and whether it has caused dysfunction at other nodes in the network (Kaiser and Hilgetag, 2004; Honey and Sporns, 2008; Alstott et al., 2009). If at a centralized location, symptoms may be more severe and reflect distributed effects than when the lesion is less centralized, in which case the effects may be largely attributed to the specialized function of the ischemic area (Alstott et al., 2009). Accordingly, recovery of function may depend on the repair and redistribution of activity in structurally intact, yet functionally disconnected nodes of a taskrelevant network.

In this review, we aim to explore the subject of functional connectivity, defined as temporal correlations between neural or hemodynamic signals arising from distinct brain regions. We focus on functional connectivity in relation to motor recovery after stroke by providing a synthesis of findings that highlight three important points. First, an ischemic stroke lesion causes disruptions in functional connections to areas remote to the site of the lesion. Second, the intrinsic architecture of the residual functional connections reflects the behavioral consequences of stroke. Third, reorganization within a functional network is possible and plays a key role in the recovery of motor function. We begin with a description of current analysis techniques used to assess functional connectivity after stroke. We then discuss functional networks in a stroke population 
as they relate to anatomical substrates, motor task performance, and resting-state paradigms. The final sections shed some light on concerns specific to the relationship of functional connectivity with motor recovery after stroke as well as suggestions for future research. Because motor recovery of the upper extremity is most commonly evaluated in neuroimaging studies, we focus here on the recovery of sensorimotor arm and hand function.

\section{TECHNIOUES FOR ASSESSMENT OF FUNCTIONAL CONNECTIVITY}

The synchronous firing of transient neural signals is what functionally binds widely distributed sets of neurons (Singer, 1999). Currently, there is no consensus on the most accurate method of assessing functional connectivity, but in many respects, the temporal resolution (milliseconds) of electroencephalography (EEG) and magnetoencephalography (MEG) is optimal for non-invasively capturing the precise timing of this activity. Changes in the electromagnetic field related to neuronal activity can be acquired over a broad frequency spectrum and spectrally decomposed into distinct frequency bands. Measurements of "coherence" or "phase synchronization" are two techniques that are specific to these two modalities and may be employed to depict the functional coupling between neural populations. Coherence is a technique that evaluates the covariance of the phase and amplitude of oscillations while phase synchronization assesses the precision of neuronal discharges independent of amplitude. Functional connectivity analyses of EEG and MEG data is sometimes performed at the level of sensor correlations which can be difficult to interpret in terms of the underlying brain structures involved. More recently, studies have examined correlations between inferred brain activity obtained by solving the MEG/EEG inverse problem. While these studies allow us to interpret the functional connectivity of underlying brain structures involved, caution must be placed on these findings due to potential confounding effects resulting from the reconstruction algorithms used.

Evaluations of coordinated neural activity have also been derived from covariations of the amplitude and latencies of hemodynamic signals arising from distinct brain regions. Techniques such as fMRI and PET make indirect inferences about neural activity through recordings of alterations in blood oxygen level dependent (BOLD) signals or metabolic activity. In an ischemic brain, the hemodynamic underpinnings of these signals may be confounded and must be cautiously interpreted and appropriately modeled through advanced statistical techniques. Still, in many cases it is possible to define functionally connected nodes within a widespread neural network located remote to the site of the lesion.

The two most commonly applied techniques to measure functional connectivity following stroke are seed-based correlation and component analysis, which can capture both resting and task-based activity in the brain. Seed-based approaches were first introduced by Biswal et al. (1995), who correlated the mean time course of the BOLD signal within a region of interest (in this case, left motor cortex) with the time courses of all other brain voxels. The results indicated correlated brain activity between bihemispheric primary and secondary sensory and motor areas and supplementary cortex even in a resting brain state. To explain this phenomenon, Biswal proposed that the fluctuations in blood oxygenation or flow is a result of functional connectivity between these regions (Biswal et al., 1995). A limitation of this technique, however, is that a priori data assumptions are required to define the seed region, which may introduce selection bias. This point becomes particularly relevant following stroke when the focal lesion may cause widespread network changes that can no longer be defined by expected normal distributions. As an alternative to seed-based connectivity, independent component and principal component analysis techniques have been applied (Friston et al., 1993; Calhoun et al., 2001; Beckmann et al., 2005). These statistical approaches evaluate the extent of signal covariance between all voxel (volume element) pairs for the entire voxel matrix of brain space. The resulting set of components account for independent (orthogonal) amounts of variance in the observed data. In terms of functional connectivity, each component represents a spatially distinct functional neural network that is highly intercorrelated.

Other, more advanced techniques that are gaining recognition as a meaningful indices of connectivity are based on graph theory. Within this framework, information processing and propagation is topographically represented by a set of nodes (i.e., brain regions) and links between the nodes (i.e., functional connections; Bullmore and Sporns, 2009). A variety of metrics have been proposed to quantify network structure based on graph theoretic criteria (Gerloff and Hallett, 2010). For instance, networks can be described as either regular, small world, or random based on the number of local connections at each node and the path length between nodes. In general, a small world may be considered optimally efficient, having a high clustering coefficient (i.e., number of local connections) with some long-range connections. On the other hand, a random network demonstrates less clustering and more long-range connections. Thus, by virtue of the distance in which most information must travel, random networks are considered relatively inefficient. The degree of efficiency is one metric that has been used to quantify brain networks and is defined by an inverse relationship with path length (i.e., distance required to go from one network node to another) and a positive relationship to clustering coefficients. By this measure, brain networks are found to be small world. Another popular graph theoretic metric is the degree of centrality. Nodes with a high degree of connections linking it to other nodes of the network are referred to as having high centrality, which elevates the importance of the hub within the overall network. Graph theoretic methods allow us to quantify network structure. While currently many of these measures describe global properties in brain networks, more efforts should be aimed at quantifying localized metrics as a means of probing local network structure.

Techniques described thus far represent functional connectivity and must be distinguished from a more recently applied subset of techniques known as effective connectivity. While functional connectivity represents two connected regions, effective connectivity depicts the intrinsic or task dependent influences that a particular area exerts over another. Two statistical methods of effective connectivity have been employed to evaluate the impact of stroke lesion and subsequent reorganization patterns (Penny et al., 2004). Dynamic causal modeling is one approach, whereby the brain is treated as a deterministic system in which known external inputs cause changes in neural activity (Marreiros et al., 2008). Accordingly, 
neuronal activity is explicitly modeled using direction and timing information. Structural equation modeling is the second technique and is similar in approach to dynamic causal modeling in that it confirms how well a model fits the data, but structural equation modeling assumes that the interactions are instantaneous or driven by an unknown source (Penny et al., 2004). Because only a select number of regions can be included for either dynamic causal modeling or structural equation modeling, it is important to keep in mind that changes may exist in regions outside of the pre-defined model (Mechelli et al., 2002).

Finally, while not considered a measure of functional connectivity per se, transcranial magnetic stimulation (TMS) approaches have been employed to probe intra- and intercortical physiology and causal changes in the intrinsic circuits in the brain (Reis et al., 2008). In a stroke population, recent attention has been directed toward the notion of interhemispheric inhibition (IHI) leading from the contralesional to the ipsilesional motor cortex (Dimyan and Cohen, 2010). Within this context, a paired pulse technique is used, whereby a test stimulus over the ipsilesional cortex is preceded by a suprathreshold conditioning stimulus to the contralesional motor cortex. Electromyographic (EMG) electrodes over the muscle of interest then record the resulting modulation of, what is known as, a motor evoked potential (MEP). Another approach used to assess connectivity is known as "jamming" or "virtual lesion," in which trains of repetitive TMS (rTMS) can used to inhibit activity arising from a pre-defined region, such as contralesional M1 or premotor cortex (Johansen-Berg et al., 2002; Lotze et al., 2006). The behavioral effects are simultaneously measured during motor task performance. Therefore, rather than a direct measure of connectivity, inferences about the influential state of cortical connections may be evaluated using TMS. One limitation, however, is that since current is reduced as a function of distance from the TMS stimulation coil, resulting brain stimulation and subsequent IHI or regional inhibition is generally restricted to the cortical level. Also, as with structural equation modeling and seed-based functional connectivity, interpretation of TMS observations only go as far as the pre-defined areas of stimulation/inhibition. By incorporating a thorough understanding of the anatomical underpinnings and important nodes of a functional network, TMS offers an immense potential to provide further insight into brain connectivity and to better guide rehabilitation efforts following stroke.

\section{ANATOMICAL SUBSTRATES OF FUNCTIONAL CONNECTIVITY}

It is widely assumed that, for the most part, functional connections reflect neuro-anatomical substrates (Fransson, 2005; Greicius et al., 2009; Honey et al., 2009; van den Heuvel et al., 2009). The strongest correlations of oscillating neural signals are thought to exist between structurally connected regions, while weaker functional ties reflect indirect structural connections (Honey et al., 2009). However, one crucial point to keep in mind is that the nature of the relationship between functional and structural connectivity is far from clear. Current methods used to quantify disrupted neural connections are anatomical tracings such as diffusion tensor imaging (DTI) in conjunction with tractography and high angular resolution diffusion imaging (HARDI) with residual bootstrap q-ball fiber tracking (Stinear et al., 2007; Berman et al., 2008; Schaechter et al., 2009). These techniques provide information about the passive diffusivity of water molecules, which preferentially travel parallel to white matter axonal fiber bundles. Despite the remarkable contribution of these approaches to the recreation of axonal networks within the brain, an ongoing limitation is the uncertainly by which a tract can be delineated (Chung et al., 2006). Water molecules inevitably encounter crossing, merging, or kissing fibers throughout the brain, thereby leading to false positives and false negatives when defining the principal diffusion direction of a given fiber tract. This limitation is particularly true with DTI fiber tracking techniques as opposed to q-ball deterministic or probabilistic tracking that specifically accounts for crossing fibers. Moreover, a common assumption in attempting to predict functional from structural connections is that the relationship will be one to one. Certainly, a direct relationship is possible, but functional connections may also be characterized as one to many or even many to one, which are very difficult to structurally resolve considering that many of these connections involve small and difficult to discern intra- and intercortical connections. Also, because tractography assesses the probability of finding a tract rather than the strength of the connection, results cannot be directly compared to the resulting functional connections, which are defined by strength. Nonetheless, while acknowledging these limitations, there still exists a pronounced need for more directed studies aimed at resolving the implied structure-function relationship in an integrated, multimodal approach. In subjects with stroke, these techniques have mostly been assessed in parallel, which underscores the difficulty in linking the two modes of neural connectivity as a comprehensive depiction of recovery.

To support and understand interhemispheric functional connections apart from DTI techniques, studies of the corpus callosum - the largest connective structure in the brain - provide evidence of a feasible anatomical substrate. While there has been considerable debate on whether the interhemispheric transfer of information is excitatory or inhibitory in healthy controls, the general consensus is that most connections between the two primary motor cortices are inhibitory to maximize the segregation of crosscortical activity (Ferbert et al., 1992). Another intriguing postulate is that the inhibitory and facilitatory nature of the transcallosal connections may differ depending on the particular dynamics of the task demand. For example, TMS studies of healthy controls found motor preparation to initially be accompanied by inhibition, followed by facilitation immediately prior to movement onset (Murase et al., 2004). Following stroke, the interhemispheric balance may be altered, such that both movement preparation and execution are accompanied by an abnormal persistence of intracortical inhibition on ipsilesional M1 (Murase et al., 2004; Hummel et al., 2009). One question currently being pursued is whether these inhibitory mechanisms are interfering with recovery rather than helping it as was found in some patients with subcortical lesions and moderate to good recovery (Murase et al., 2004; Hummel et al., 2009). As an alternative hypothesis, the contralesional M1 and premotor cortex may be a potentially relevant substrate for recovery. Using single pulse TMS, Johansen-Berg et al. (2002) demonstrated the importance of contralesional premotor cortex in people with profound motor impairments performing a simple reaction time task. As an extension to these findings, Lotze and colleagues used rTMS and a "virtual lesion" approach to demonstrate that the control complex 
sequential finger movements in well-recovered subjects relies on the contribution of the contralesional premotor and primary motor cortex. Nevertheless, since TMS studies present a coarse perspective of network function, a better understanding the underlying of functional architecture may enhance interpretation and provide direction as to the most important regions of stimulation.

\section{TASK-BASED CONNECTIVITY}

To date, theories of the brain's response to a stroke lesion emphasize extensive changes in localized functional activation patterns during a motor task compared to that of a healthy control population. In general, the response to affected limb movement initially includes a bilateral overactivation in primary and secondary sensorimotor regions. During the process of recovery, a reduction in activation is observed that can either persist in a bilateral distribution or become lateralized toward the perilesional tissue and motor regions of the lesioned hemisphere (Calautti et al., 2001; Ward et al., 2003). Presumably, these changes represent an attempt to maximize the residual cortical output, but the involvement of multiple areas is generally inversely related to recovery. In the event that the ipsilesional primary motor cortex is no longer capable of functional contributions, secondary regions gain importance, and perhaps become a necessary component to sustain further, albeit incomplete, recovery. In this case, cortico-cortico and corticospinal tract are the two primary connections, although the specific contribution of each pathway to motor recovery is still unknown. While the identification of these localized activation patterns has been informative with respect to the recovery process, we now have the analysis tools to deepen the understanding of the underlying neural integration. That is, spatial reorganization implies an underlying network of activity, but the analysis fails to explicitly identify functional connections and, more importantly, the evolution of these connections as a process of recovery.

\section{FUNCTIONAL CONNECTIVITY}

Moving beyond investigations of the reorganization of localized neural activations, Seitz et al. (1999) was the first to probe functional network changes induced by a stroke lesion. PET data was collected in seven subjects in the chronic ( $\sim 6$ months) stage after infarction. Subjects were scanned at rest and during performance of finger movement sequences as accurately and fast as possible. Using principal component analysis, results indicated that of the eight principal components representing $80 \%$ of PET data variance, two were differentially expressed in controls and subjects with stroke. The first component, deemed, the "lesion-affected" network, was expressed in the absence of task and supported the widespread abnormalities occurring in regions remote to the ischemic core. Specific changes included the ischemic core and perilesional area, as well as contralesional and subcortical structures. The second component was expressed during finger movement and correlated with motor scores acquired within a few days of stroke. Consequently, the authors deemed this component a "recovery-related" network. Of particular interest was the spatial overlay of the lesion-affected and recovery-related networks, which demonstrated similar areas of connectivity in the thalamus and visual association areas. Because the thalamus is an area of visual processing, it is not surprising that these regions fall within an integrated network, but why they are involved in both networks remains unanswered. One intriguing explanation is that remote resting network changes induced by the lesion may, in fact, facilitate motor recovery. However, a relationship between either of these networks and motor performance at the time of imaging (i.e., chronic stage) was not demonstrated, leaving questions as to the relevancy of these connectivity patterns to motor impairments and the process of recovery. Nevertheless, this early study highlighted the potential usefulness of a functional network approach, beyond localization theories, to identify disrupted brain activity following stroke.

Electroencephalography coherence studies during affected hand movement have also shown changes in the patterns of cortical interactions compared to healthy controls, yet still with limited relationship with motor performance. For instance, Strens et al. (2004) recorded brain activity from nine electrodes in 25 subjects in the chronic (1-6 years) post-stroke stage during a $25 \%$ maximal handgrip task. Corticocortical coherence was determined in the combined alpha and low beta-frequency bands $(9-25 \mathrm{~Hz})$ and averaged across six connectivity groupings: left lateral frontal (three electrode pairs), right lateral frontal (three electrode pairs), left mesiolateral (nine electrode pairs), right mesiolateral (nine electrode pairs), mesial (three electrode pairs), and interhemispheric (nine electrode pairs). Of these regions, coherence was greater in subjects with stroke compared with control subjects during task execution in three connectivity patterns, namely, between ipsilesional mesial (SMA) and lateral frontal region (sensorimotor cortex), over contralesional lateral frontal region (sensorimotor cortex), and over contralesional mesial motor region (SMA). The authors speculated that increased connectivity of mesial and lateral frontal regions related to increased attention to task that, in cases of incomplete recovery, represented a compensatory mechanism. Also of note was that the group differences appear specific to the task execution since no differences were identified during the task preparation phase (Strens et al., 2004). Although these changes were interesting from a connectivity perspective, there was again a general lack of association with motor performance, which may merely reflect the limitations in the EEG coherence metric used to identify cortical changes. Specifically, the authors considered a "hand difference score" representing asymmetries in cortical coherence obtained during affected and unaffected hand movement. Therefore, group differences in the degree of asymmetry may have partially been driven by reorganization of cortical networks during unaffected hand movement as may have arisen through compensatory overuse of this hand. Consequently, interpretation of these results was somewhat confounded and required further research to isolate changes of the affected hand.

Apart from the relatively unknown association with motor performance, one particularly surprising finding of the previous study was the lack of change in interhemispheric connectivity. Given that the commonly observed increased activity of ipsilesional sensorimotor cortex is suggestive of increased output from this region, the authors conducted a follow-up study to further evaluate the interhemispheric coupling (Serrien et al., 2004). Within this study, they also chose to disentangle changes due to movement of the unaffected from those of the affected hand. Using a directed coherence approach, it was observed that, in the low beta-frequency band, information flowed from the contralesional 
to the ipsilesional sensorimotor cortex during affected hand movement in less recovered subjects. Directed information flow was also found from mesial to contralesional sensorimotor cortex, again highlighting the importance of SMA as part of a larger network. Thus, in patients with incomplete recovery, the importance of connectivity in the contralesional sensorimotor cortex, possibly acting under the influence of mesial areas is supported in this study. However, whether these integrative effects are facilitatory or inhibitory remained blurred and have since become an important focus of TMS research (Dimyan and Cohen, 2010). Also, it should be kept in mind that the inclusion criteria were primarily based on motor impairment status (i.e., weakness of wrist and finger extensors). As a result, differences in lesion location (cortical, subcortical, left, right), lesion type (ischemic, hemorrhagic), and initial level of severity may have introduced important confounding variables to the connectivity patterns and data interpretation.

In an effort to further clarify network contribution of contralesional primary motor cortex to motor recovery in a very specific patient cohort, Gerloff et al. (2006) employed a multimodal analysis. Eleven subjects with chronic ischemic lesions (1-9 years post-stroke onset) of the left internal capsule and mild to moderate extremity impairments were included. Functional connectivity was assessed using EEG corticocortical coherence during affected finger extension and interpreted using TMS MEP of ipsilesional and contralesional M1. An increase in functional coupling was observed between contralesional motor/premotor cortex and SMA in all frequency bands tested (low alpha $8-10 \mathrm{~Hz}$; high alpha $11-13 \mathrm{~Hz}$; low beta $16-20 \mathrm{~Hz}$, high beta $22-26 \mathrm{~Hz}$ ) compared to healthy controls. In contrast, reduced connectivity was found between links of ipsilesional motor/premotor cortex with SMA and contralesional motor/ premotor cortex in the low beta band. These results largely corroborate findings of Serrien et al. (2004), supporting key connections between SMA and contralesional M1 in subjects with residual motor deficits. Interestingly, TMS applied to contralesional M1 did not induce a motor response of the affected hand, essentially refuting the possibility of compensation through corticospinal commands from this hemisphere, at least in subjects with mild impairments. Gerloff et al. (2006) additionally tested the localization of functional activation using PET during affected hand movement. Without any insight other than a bilateral activation response pattern, the advantages of assessing functional connections over local activations to understand neural changes after stroke were clear. However, although there are benefits to using a multimodal approach to better inform EEG and fMRI functional connectivity, interpretation may be enhanced by considering the directionality of information flow using measures of effective connectivity.

\section{EFFECTIVE CONNECTIVITY}

Grefkes and colleagues used fMRI and dynamic causal modeling to identify the impact of subcortical stroke lesions on neural networks during whole hand flexion/extension. Subjects were assessed in the subacute (5-32 weeks) stage of recovery and each demonstrated mild weakness of their hand. During affected hand movement, inhibitory influences from contralesional to ipsilesional M1 were observed that related to the extent of motor impairment (Figure 1). Bilateral hand movements were also included and resulted in a reduction in the facilitatory drive leading from ipsilesional M1 to contralesional M1 compared to healthy controls. Moreover, a reduced facilitation was demonstrated from ipsilesional SMA to contralesional M1 that correlated with bilateral hand motor deficit. Even in the absence of task performance, differences in interhemispheric coupling between both SMAs and ipsilesional coupling between SMA and M1 were identified and were suggested as further mechanisms underlying hand motor impairment (Grefkes et al., 2008). While these results corroborated previous TMS studies of IHI during a unimanual motor task, dynamic causal modeling enabled the assessment of the motor network beyond M1. As stated by the authors, these findings may have important implications for treatment. If bilateral arm activities can improve the facilitatory effect of SMA, then promoting activity of contralesional M1 may, in turn, facilitate ipsilesional M1 as seen in healthy controls. Presumably, the facilitatory effect would extend to unimanual movements of the affected hand. Further longitudinal interventions are required to support these hypotheses.

In addition to effective connectivity analysis of the classical motor network, the inclusion of cognitive regions revealed new areas of connectivity after stroke during a bilateral hand task (Walsh et al., 2008). Using fMRI and structural equation modeling, interhemispheric connectivity of prefrontal cortex with ipsilesional SMA was observed in subjects with stroke that was not apparent in healthy controls, suggesting that attention to action or explicit learning may be a key compensatory mechanism following stroke. Clinical implications may initially include the promotion of this mechanism to facilitate SMA and, through interhemispheric connectivity, ipsilesional M1. Later, as recovery ensues, movement may become more implicit such that the activation is again limited to the classical motor network, in the absence of prefrontal input.

Recognizing a need to identify whether pathological changes in connectivity may be reversed through targeted TMS interventions, Grefkes et al. (2010) assessed subjects using fMRI and effective connectivity analysis immediately before and after paired pulse TMS stimulation. Dynamic causal modeling revealed that TMS did indeed induce interhemispheric network changes resulting in reduced suppression from contralesional to ipsilesional M1 during a unimanual affected hand task. These network changes were observed in relation to enhanced motor performance. A bilateral task was additionally evaluated, but because of an overall positive coupling between all motor regions during a bilateral task, network changes during bimanual movements were not observed. In other words, a suppression of contralesional M1 was not indicated in this model and therefore, was not expected to induce changes (Grefkes et al., 2010).

Effective connectivity changes within an extended motor network have also been revealed during motor imagery following stroke. Sharma et al. (2009) evaluated the extent to which motor recovery is associated with network reorganization induced by motor imagery compared to motor execution. fMRI and structural equation modeling were assessed in subjects who were 1 week to 2 years post-stroke. Results revealed differential connectivity patterns in motor imagery and execution networks between subjects with stroke and controls. Specifically, a reduction in the connectivity between ipsilesional SMA and premotor cortex was found in both networks, while only during motor imagery was increased coupling observed between ipsilesional 


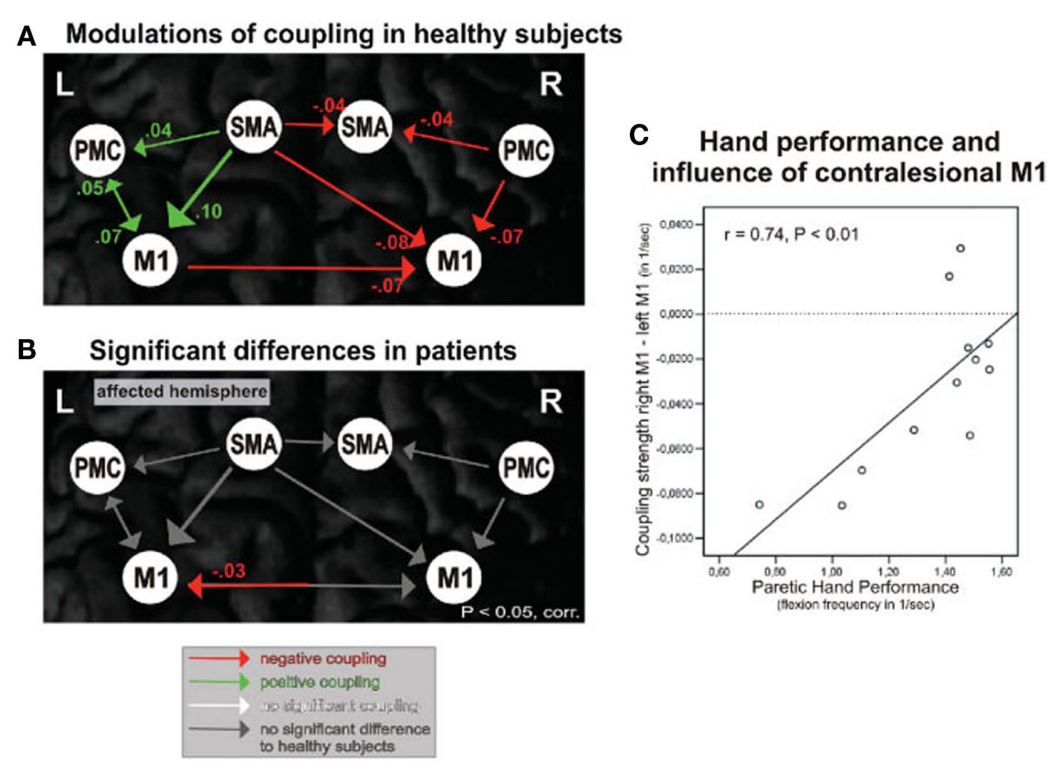

FIGURE 1 | Effective connectivity of motor network during unimanual hand movements. (A) Functional coupling in healthy control subjects during right hand movement. (B) Significant differences in functional coupling during affected (right) hand movement between healthy control subjects and subjects with stroke. (C) Significant correlation between rate of affected hand movement and strength of inhibitory connections from contralesional M1 to ipsilesional M1. SMA, supplementary motor area; PMC, premotor cortex; M1, Primary motor cortex. Reproduced with permission from Grefkes et al. (2008). prefrontal and both premotor cortex and SMA. These results are in line with previous work demonstrating the importance of premotor cortex to stroke recovery (Johansen-Berg et al., 2002) and the inhibitory coupling between SMA and M1 in healthy controls (Solodkin et al., 2004). In terms of motor performance, only connectivity within the motor imagery network, and not the motor execution network, was relevant. That is, positive correlations were observed between hand function (assessed following motor imagery) and connectivity from contralesional prefrontal cortex to SMA as well as from ipsilesional prefrontal cortex to premotor cortex. Therefore, increased connectivity with prefrontal cortex may have also facilitated recovery. Negative correlations were identified with connectivity between ipsilesional SMA and premotor cortex. The authors contend that abnormal integration of the prefrontal cortex within the motor network in patients with stroke is due to the important role of this region in motor preparation and planning. In particular, the cognitive rehearsal of the motor planning program that inevitably takes place during motor imagery may be the reason for the increased connectivity in this population. Once again, it should be mentioned that differences between subjects with stroke and controls were not observed using classical analysis of changes in localized functional activations for either task (motor imagery or execution), thereby reinforcing the importance of evaluating motor network changes (Sharma et al., 2009).

In general, the interpretation of the combined findings of these studies is that lesions in one hemisphere cause dysfunctional connections with contralesional motor regions that appear to mediate unimanual and bimanual impairments during hand motor tasks. Evidence is in support of the importance of secondary motor regions, particularly SMA, that influence ipsilesional M1, either directly or indirectly through contralesional motor cortex or ipsilesional premotor cortex. Whether the influence of this region is compensatory due to enhanced attention to task mechanisms or marks true reorganization of the motor network has yet to be resolved.

Despite the potential usefulness of task-based activation studies, at least two important limitations must be considered. First, subjects are typically selected because they are able to perform a standardized task, thereby limiting the generalizability of findings to the function of the particular task and to subjects with moderate to good motor recovery (Dong et al., 2006; Calautti et al., 2007). Second, mirror movements and increased effort are two common occurrences during affected limb movement leading to exaggerated activation of the unaffected hemisphere (Wittenberg et al., 2000; Ward et al., 2007). Although it is clear that lesions induce broad changes within a cortical network or activity, the dependency on task performance in the previously mentioned studies leaves critical uncertainties of the behavioral link. Are these network changes representative of the recovery process and cortical reorganization after stroke or are they merely an epiphenomenon?

\section{RESTING-STATE CONNECTIVITY}

Resting-state functional connectivity represents a reliable and promising means of assessing the intrinsic transfer of neural information within a network while avoiding many task-based confounds (Damoiseaux et al., 2006). Although the physiological source of spontaneous activity is unclear, validation studies using fMRI and EEG identified a spectral profile of rhythmic neural activity within a number of functionally relevant networks. For example, neural oscillations within a combination of primarily low frequency bands was found to contribute to the BOLD signal contributing to a sensorimotor network (Mantini et al., 2007; Nir et al., 2008). Moreover, using MEG, regions identified as having the highest density of functional 
connections in the alpha "idling" frequency band reflected the high functional demands placed on these regions during daily tasks including somatosensory, visual, and language cortices (Guggisberg et al., 2008). Clearly, because this testing paradigm is not induced by an active task, it allows for an assessment of functional connectivity within multiple neural networks collected during a single experimental session. Equally important is the possibility to overcome many of the limitations of task-based paradigms, particularly as they relate to stroke as mentioned in the previous section. Such intrinsically connected networks have also been proposed as useful biomarkers of sensorimotor impaired brain states such as Parkinson's disease (Stoffers et al., 2008; Wu et al., 2009), amyotrophic lateral sclerosis (Mohammadi et al., 2009), multiple sclerosis (Lowe et al., 2002; Cover et al., 2006), and brain tumors (Guggisberg et al., 2008). Because the concept of resting-state networks as they apply to neurological conditions is still relatively new, only a handful of studies have employed this method to assess the influence of stroke. However, the consensus within these studies is that resting-state connectivity is indeed disturbed by a stroke lesion and the resulting spatial patterns of connectivity are related to functional outcomes.

The sensorimotor resting network, in particular, has proven to be important in understanding motor deficits. Carter et al. (2009) used seed regions within an attention and motor network to evaluate interhemispheric and intrahemispheric connectivity in relation to clinical motor deficits in an acute (9-31 days) stage after stroke. Regions of the motor network included bilateral sensorimotor cortex, SMA, secondary somatosensory cortex, putamen, thalamus, and cerebellum. Results revealed a disruption to interhemispheric functional connectivity of homologous pairs within both networks that correlated with upper extremity impairment. Remarkably, intrahemispheric connectivity, even within the lesioned hemisphere, did not relate to behavioral outcomes. Also, although the focus of this review is on upper extremity impairments, it is worth mentioning that lower extremity deficits and gait impairment were most highly correlated with the attention network rather than the sensorimotor network. This finding is well in line with the clinical observation of the marked difficulty in attending to a secondary task while walking. Thus, the critical point in this study was that the strength of cross-cortical functional connections assessed in the resting brain are related to motor tasks. Equally important is that these results could not be explained by structural damage, since for the most part, the attention and motor networks were outside of the lesion core (Carter et al., 2009).

While the strength of connectivity is one characterizing feature of resting-state functional connectivity, pathological changes may also be quantified by the degree of efficiency in which information flows between connected regions. In a recent longitudinal study, Wang et al. (2010) described dynamic changes in network efficiency using a graph theoretical approach. $\mathrm{AMRI}$ data was collected at five post-stroke time points ( 1 week, 2 weeks, 1 month, 3 months, and 1 year). The primary finding was that changes occurring within a motor network, defined by 21 brain regions, progress toward a random, less optimized network (Figures 2A,B). That is, nodes tend to become less clustered and information must travel over longer distances as more time passes from the stroke event. Interestingly, the degree of randomization positively related motor recovery. Though the underlying mechanisms of this process remain unclear, the authors site the possibility of changes occurring on a cellular level that are known to include a random outgrowth of new axonal connections (Kaiser et al., 2009). Regardless of the cause, however, a shift toward random networks is not new to brain pathology and has been shown before in the presence of brain tumors (Bartolomei et al., 2006), Alzheimer's disease (de Haan et al., 2009; Stam et al., 2009), epilepsy (van Dellen et al., 2009), and traumatic brain injury (Nakamura et al., 2009). Therefore, as the authors of this study state, network randomization may represent a final common pathway for many brain pathologies when normal connections are impaired. Further investigations are required to better understand this phenomenon and whether randomization is indeed facilitating recovery or merely hindering the potential for further recovery.

Along with network efficiency changes, the emergence of asymmetries in the importance of centralized "hubs" of connectivity within a motor network appear to be related to the recovery process after stroke. As time post-stroke progresses, Wang and colleagues also report that the greater the degree of centralization within ipsilesional M1 and contralesional cerebellum (dentate nucleus), the better the clinical outcomes, while the inverse was true of the ipsilesional cerebellum and thalamus (Figure 2C). Compared to controls, specific regions to which ipsilesional motor cortex demonstrated increased connectivity included contralesional motor cortex, post-central gyrus, and ventrolateral premotor cortex, as well as bilateral dorsolateral premotor cortex. Contralesional cerebellum revealed increased connectivity with ipsilesional ventrolateral premotor cortex. In contrast, regions to which ipsilesional thalamus demonstrated reduced connectivity included bilateral dorsolateral premotor cortex and basal ganglia as well as contralesional SMA. Ipsilesional cerebellum was less connected with contralesional cerebellum and bilateral basal ganglia. Importantly, the notion of increased connectivity within key regions may have critical implications for prognosis and treatment (Wang et al., 2010).

Lesions identified within the above-mentioned centralized hubs of connectivity create models in which lesion location is of high importance and may offer a good predictor of recovery potential. In a sophisticated computational model of known structural connections, Alstott et al. (2009) deleted nodes based on centrality within the theoretical network and effectively predicted non-local brain effects. For example, deletion of nodes within regions of the frontal cortex resulted in particularly large and widespread effects, whereas lesions directly over primary sensory or motor cortices induced smaller, but primarily interhemispheric as opposed to intrahemispheric, disruptions (Figures 3A,B). One consideration in employing a network model to predict recovery is the time in which connectivity changes develop, which may depend on the delayed spread of local neural disruption from the lesion site. For instance, the randomization noted by Wang et al. (2010) did not begin to emerge until 10-14 days after stroke, suggesting that a certain amount of time must elapse before the deterioration and reorganization of connections leading to and from remote regions may be statistically captured and used to predict outcomes. This timeline is in contrast to previous stroke predictive markers based on early identification of penumbral size and the extent of Wallerian degeneration of the corticospinal tract (Witte et al., 2000; DeVetten et al., 2010). Since it is well known that early stroke deficits are not 

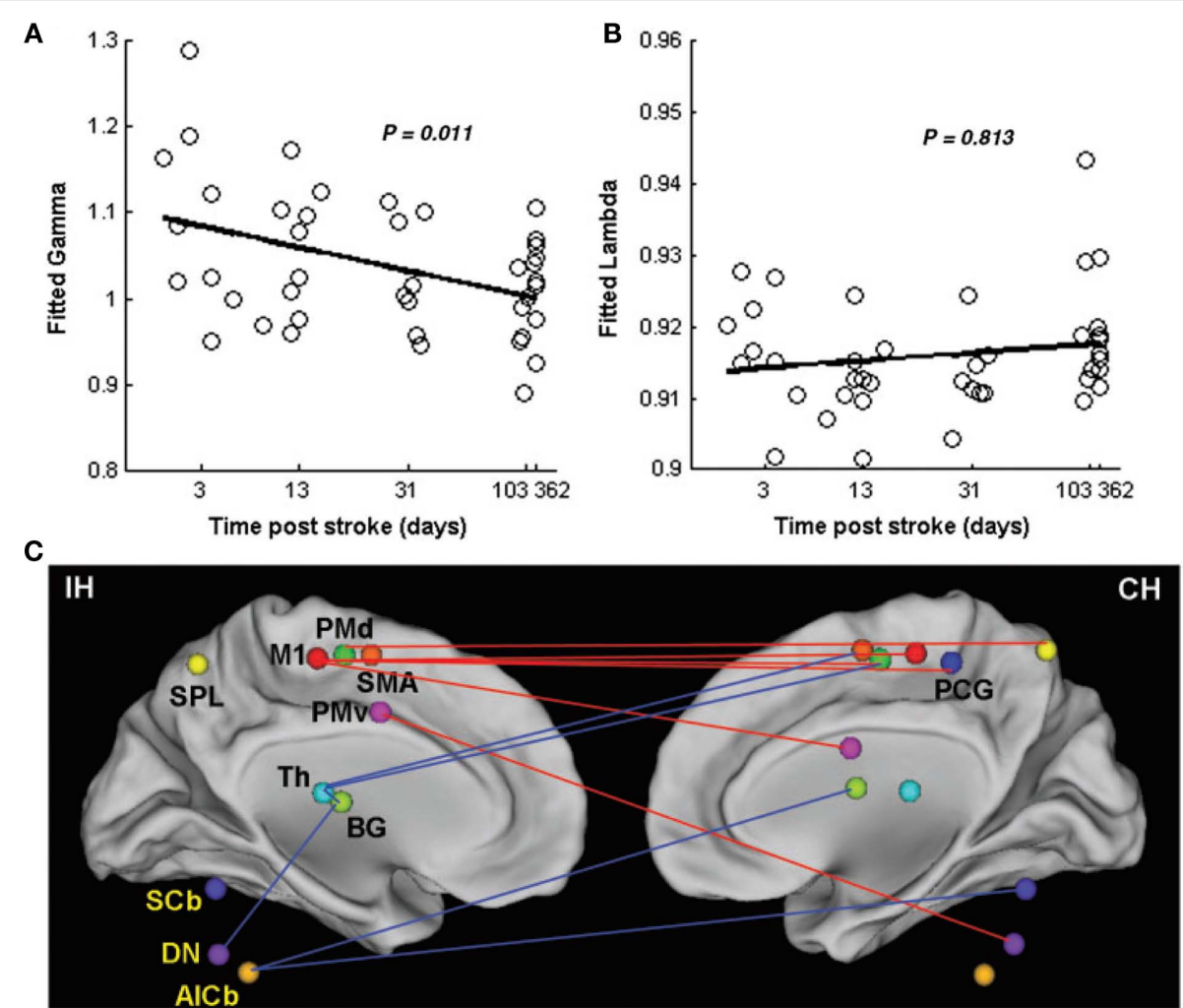

FIGURE 2 | Changes in network efficiency over time post-stroke. (A) Significant negative correlation between clustering coefficients (fitted gamma) and time post-stroke represents shift toward a random network configuration. (B) Non-significant positive correlation between the shortest path length (fitted lambda) and time post-stroke (C) Connectivity parameters between nodes of the motor network. Increased connectivity (red lines) are primarily seen as interhemispheric connections between $\mathrm{M} 1$ and contralesional sensorimotor regions. Reduced connectivity (blue lines) is mainly found in ipsilesional subcortical areas and cerebellum. $\mathrm{IH}$, ipsilesional hemisphere; $\mathrm{CH}$, contralesional hemisphere; M1, primary motor cortex; PCG, post-central gyrus; PMd, dorsolateral premotor cortex; PMv, ventrolateral premotor cortex; SMA, supplementary motor area; Th, thalamus; BG, basal ganglia; SPL, superior parietal lobule; SCb, superior cerebellum; DN, dentate nucleus; $\mathrm{AlCb}$, anterior inferior cerebellum. Reprinted with permission (Wang et al., 2010). representative of eventual outcomes, it may be that the delays in network changes are at least one of the mechanisms accounting for further damage or repair.

Because functional resting-state networks appear to represent the foundation of neural activity, the possibility to induce change through rehabilitation is an intriguing concept. In a recent study, James et al. (2009) used fMRI and structural equation modeling to assess whether a novel intervention could alter the driving influences of connectivity within a resting motor network in subjects in the subacute stage (34-55 days) after stroke (Figure 4). The intervention followed a task specific training protocol including repetitive, goal-directed movements of the affected upper extremity at a dose of $2 \mathrm{~h}$ per day, 5 days per week, for 3 weeks. The structural equation modeling analysis included anatomically defined regions of bilateral primary motor cortices, lateral dorsal premotor cortices, and SMA. Following treatment, an increased influence of ipsilesional premotor cortex on contralesional premotor cortex was observed that positively related to improvements in motor performance. In contrast, a control subject with stroke who did not receive the intervention, demonstrated the reverse pattern: contralesional premotor cortex influenced ipsilesional premotor cortex. An intrahemispheric influence of ipsilesional premotor on M1 was also found in four subjects within the intervention group. Interestingly, the two subjects who improved the most as a result of the intervention were the only subjects to demonstrate an increased influence of SMA on ipsilesional premotor cortex, which then indirectly influenced ipsilesional M1. Although the different types and location of stroke included in this study may have confounded the results, these findings highlight the potential for asymmetrical reorganization to occur in relation to recovery after stroke rather than a return to the symmetrical connections seen in healthy controls (James et al., 2009).

As with task-based paradigms, limitations in resting-state approaches also exist. For example, recent TMS data with simultaneous motor task suggest that network activity is modulated from rest to executed movement. At this point, it remains unclear whether these changes are comparable between subjects with stroke and healthy controls. For resting-state analyses, these findings open several questions that have yet to be resolved. That is, precisely what information can we gain from resting-state activity that extends beyond knowledge of structural anatomy? How is "resting-state" defined? What is the consistent set of instructions that should be provided to all subjects (i.e., "Be free of thought?" "Eyes open or closed?"). Until a consensus is reached on the resting-state paradigm, comparisons across studies must be cautiously interpreted. 


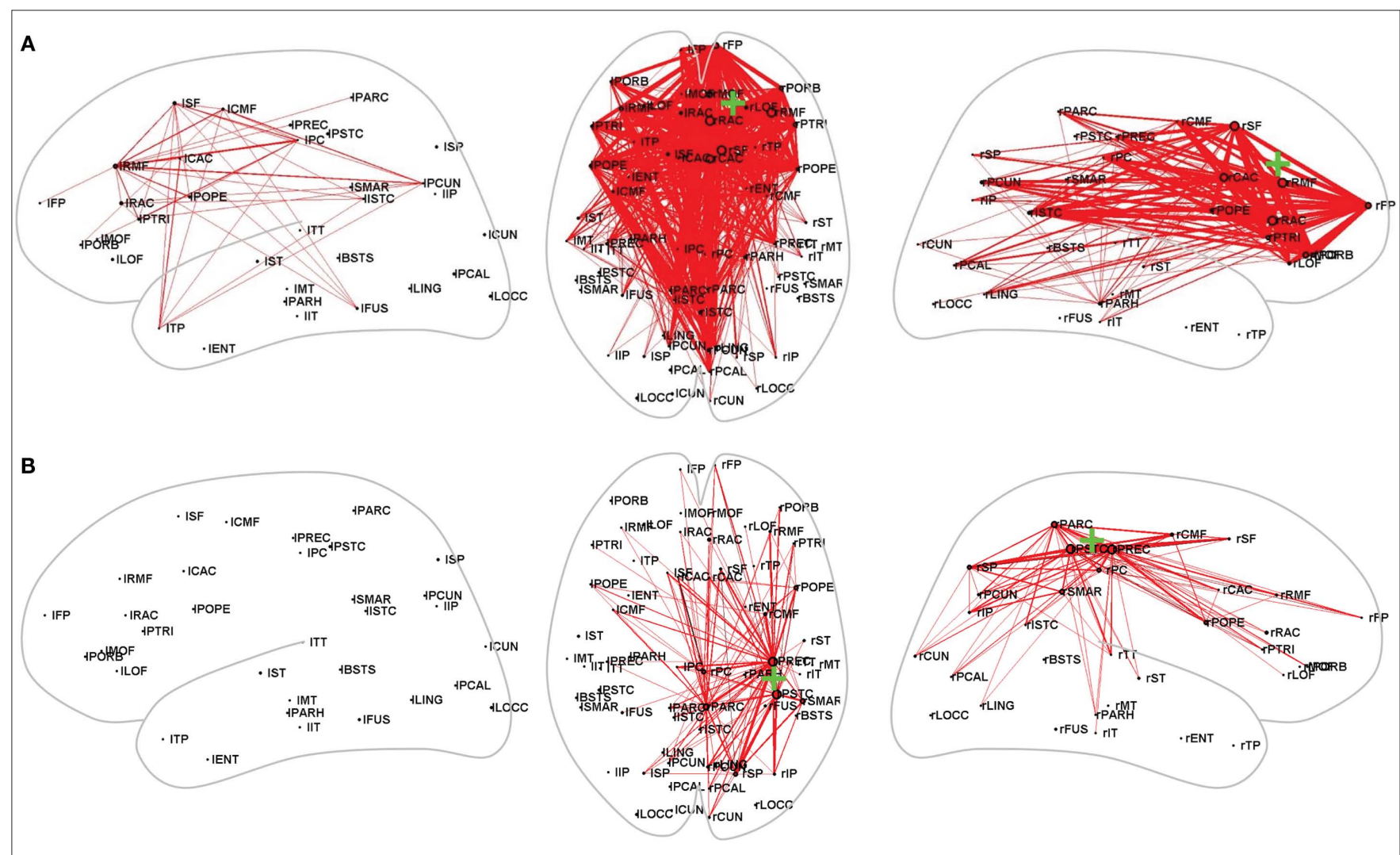

FIGURE 3 | Computational modulations in connectivity resulting from lesions in (A) Frontal cortex and (B) Sensorimotor cortex. Red lines indicate increased strength in connectivity. Note the widespread disruption caused by a lesion in the prefrontal cortex compared with the relatively constrained, intrahemispheric changes resulting from a lesion of the sensorimotor cortex. Reproduced with permission from Alstott et al. (2009).

\section{NOTES ON MEASUREMENT OF RECOVERY}

One of the surprisingly understated considerations in any study of the neural substrates of stroke recovery is the definition of recovery. Clearly, the choice of the clinical outcome by which recovery is judged and, in turn, used to interpret changes in brain connectivity patterns is tremendously important. Unfortunately, a familiar limitation of clinical outcome scales is that they often do not capture true motor recovery inasmuch as they capture compensatory changes at the behavioral level (Levin et al., 2009). To overcome these limitations and to generate an overall impression of recovery, it is recommended that either a composite score based on several outcome measures (Ward et al., 2003) or kinematic and kinetic variables should be employed. Moreover, since hemiparesis is generally more severe in the distal musculature, and less severe in the proximal musculature (Colebatch and Gandevia, 1989), evaluation of recovery should consider hand movement in isolation (i.e., grip) and in combination with proximal upper extremity movement (i.e., reach). In doing so, differential recovery of both grasp and reaching, if present, will be captured. If clinical scales are used, redundancy can be avoided by including data reduction techniques such as principal component analysis (Strens et al., 2004; Chouinard et al., 2006). For example, Strens et al. (2004) assessed subjects using a range of eight outcome measures. A principle component factor analysis revealed that only two principal components were required to adequately describe $82 \%$ of the total variance. The first component was evenly distributed across the eight outcome measures, reflecting global disability. The second component was found to reflect scores describing hand and upper limb function (Strens et al., 2004). In general, during these early investigations of strokerelated recovery patterns, the challenge will be to adequately model motor performance to best derive meaning from the relationship with functional connectivity. Just because behavioral correlations of a selected group of outcomes did not correlate does not mean a behavioral relationship does not exists with other, potentially more meaningful motor outcomes.

Loss of motor control of the so-called "unaffected" upper or lower extremity should also be considered. Given that a lesion may affect a bilaterally distributed motor network, it seems plausible that the ipsilateral limb may be affected. A handful of studies have evaluated this possibility with kinetic and kinematic measures and demonstrated bilateral impairment in grasp following unilateral subcortical stroke (Quaney et al., 2005; Nowak et al., 2007). At least two possible mechanisms account for these bilateral deficits. The first is that a subcortical lesion may damage the small percentage of ipsilesional descending corticospinal tract fibers. The second possibility is that a unilateral lesion-affected the inhibitory balance between the two homologous motor cortices. Consequently, these deficits must be taken into account when evaluating and interpreting the behavioral correlates of changes in widespread brain networks. 


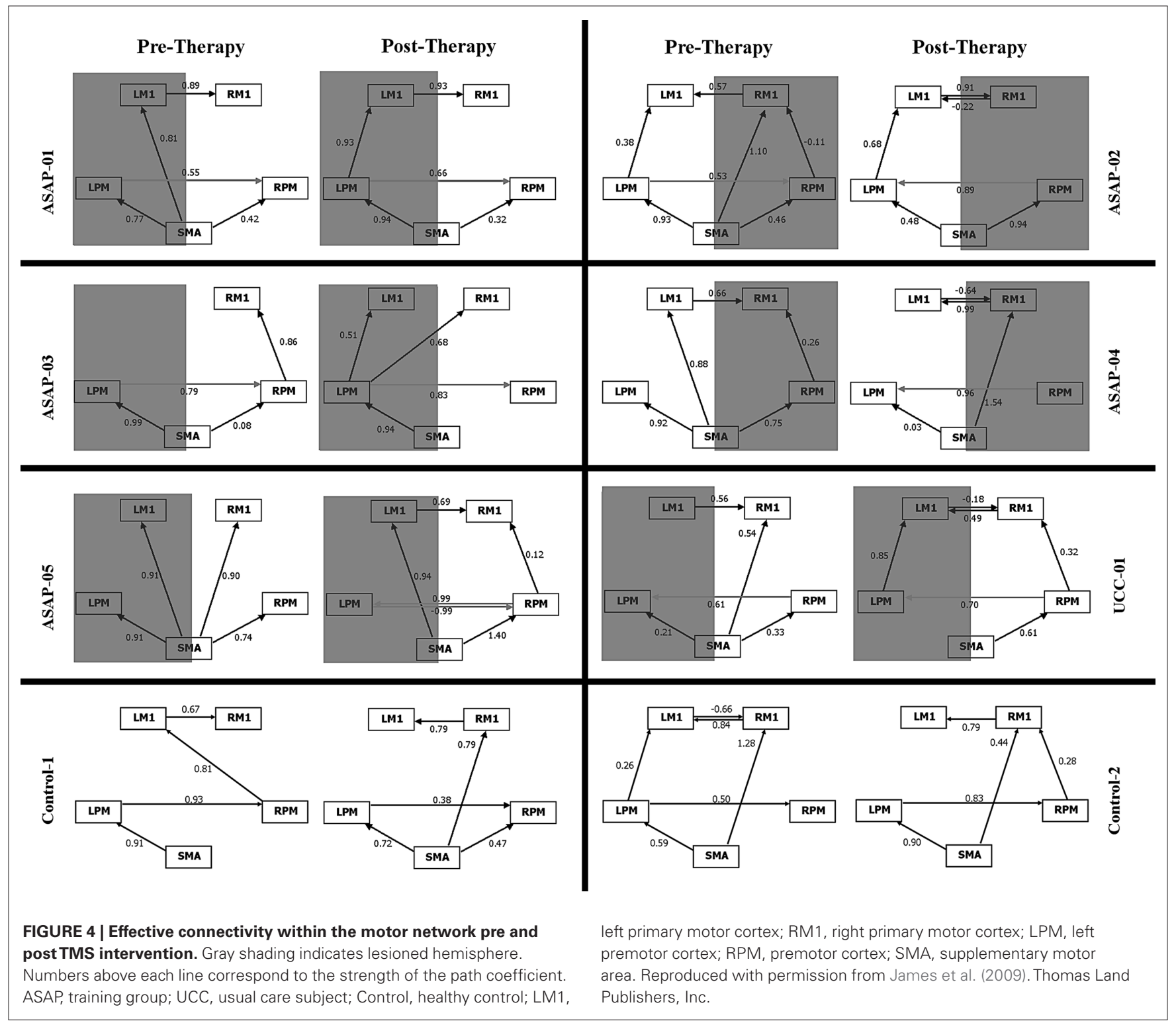

Besides adequately defining clinical outcome, capturing motor recovery also necessitates that a change in motor skill is measured. Therefore, while cross sectional designs are useful to determine the association between patterns of neural connectivity and the extent of motor control, only a longitudinal assessment will truly define the changes in neural connectivity associated with recovery. This point has been made clear in studies evaluating focal activation data (Ward et al., 2003) and now needs to be applied to connectivity data.

Finally, when evaluating functional networks during task performance across a group of subjects or at sequential time points, it is essential to control the amount of effort exerted during the task. For example, asking patients and control populations to reproduce a small percent of their maximal effort will help to match the initial performance level of all subjects. Otherwise, a fixed maximal level of exertion will inevitably overestimate changes associated with recovery (Ward et al., 2007).

\section{WHAT IS NEXT?}

The distributed impairments of connected neural systems after a stroke lesion will likely have widespread implications for clinical neuroscience. Clearly, with the relatively sparse number of studies evaluating stroke recovery from a neural network perspective, more research is needed. At this point, it remains unclear whether differences in functional connectivity between control subjects and subjects with stroke are related to adaptive or maladaptive brain reorganization, motor performance compensation, or if they are merely an epiphenomenon such as the release of the contralesional hemisphere from suppression of the ipsilesional hemisphere (Strens et al., 2004).

Overall, with a growing body of literature to support a functional connectivity approach, it is crucial that future research continue to expand the understanding of the spectrum of changes occurring in the brain after stroke. In this capacity, investigations of lesioninduced network plasticity are anticipated to challenge current 
frameworks of post-stroke rehabilitation such that patient stratification may be enhanced to maximize motor performance. Current models of stroke intervention are primarily based on behavioral status. Within the proposed recovery framework, predictions of a patient's capacity to achieve motor gains after stroke will instead be based on a more precise understanding of the neurophysiological underpinnings and, in turn, present a more accurate portrait of recovery. Unique advantages and limitations of individual imaging approaches suggest a multimodal assessment of functional connectivity as the best approach to assess this framework. Findings may then be integrated with results of structural connectivity analysis, and even genetic factors of motor learning such as BDNF and COMT, to create a powerful combination of complementary techniques to best depict the interruption and subsequent reorganization of neural networks after stroke.

In summary, neurological deficits after a focal stroke lesion not only reflect local dysfunction at the site of injury, but are also determined by the distributed impairment of connected neural systems. To a large extent, the evidence in support of functional and effective

\section{REFERENCES}

Alstott, J., Breakspear, M., Hagmann, P., Cammoun, L., and Sporns, O. (2009). Modeling the impact of lesions in the human brain. PLoS Comput. Biol. 5, e1000408. doi: 10.1371/journal. pcbi.1000408

Bartolomei, F., Bosma, I., Klein, M., Baayen, J. C., Reijneveld, J. C., Postma, T. J., Heimans, J. J., van Dijk, B. W., de Munck, J.C., de Jongh, A., Cover, K. S., and Stam, C. J. (2006). Disturbed functional connectivity in brain tumour patients: evaluation by graph analysis of synchronization matrices. Clin. Neurophysiol. 117, 2039-2049.

Beckmann, C. F., DeLuca, M., Devlin, J.T., and Smith, S. M. (2005). Investigations into resting-state connectivity using independent component analysis. Philos. Trans. R. Soc. Lond. B Biol. Sci. 360, 1001-1013.

Berman, J. I., Chung, S., Mukherjee, P., Hess, C. P., Han, E. T., and Henry, R. G. (2008). Probabilistic streamline q-ball tractography using the residual bootstrap. Neuroimage 39, 215-222.

Biswal, B., Yetkin, F. Z., Haughton, V. M., and Hyde, J.S. (1995). Functional connectivity in the motor cortex of resting human brain using echo-planar MRI. Magn. Reson. Med. 34, 537-541.

Bullmore, E., and Sporns, O. (2009). Complex brain networks: graph theoretical analysis of structural and functional systems. Nat. Rev. Neurosci. 10, 186-198.

Calautti, C., Leroy, F., Guincestre, J. Y., Marie, R. M., and Baron, J. C. (2001). Sequential activation brain mapping after subcortical stroke: changes in hemispheric balance and recovery. Neuroreport 12, 3883-3886.

Calautti, C., Naccarato, M., Jones, P. S., Sharma, N., Day, D. D., Carpenter,A. T.,
Bullmore, E. T., Warburton, E. A., and Baron, J. C. (2007). The relationship between motor deficit and hemisphere activation balance after stroke: a $3 \mathrm{~T}$ fMRI study. Neuroimage 34, 322-331.

Calhoun, V. D., Adali, T., Pearlson, G. D., and Pekar,J.J. (2001). Spatial and temporal independent component analysis of functional MRI data containing a pair of task-related waveforms. Hum. Brain Mapp. 13, 43-53.

Carter, A., S. V., A., Lang, C. E., Connor, L. T., Rengachary, J., Strube, M. J., Pope, D. L., Shulman, G. L., and Corbett, M. (2009). Resting state inter-hemispheric fMRI connectivity predicts performance after stroke. Ann. Neurol. 67, 365-375.

Chouinard, P. A., Leonard, G., and Paus, T. (2006). Changes in effective connectivity of the primary motor cortex in stroke patients after rehabilitative therapy. Exp. Neurol. 201, 375-387.

Chung, S., Lu, Y., and Henry, R. G. (2006). Comparison of bootstrap approaches for estimation of uncertainties of DTI parameters. Neuroimage 33 , 531-541.

Colebatch, J. G., and Gandevia, S. C. (1989). The distribution of muscular weakness in upper motor neuron lesions affecting the arm. Brain $112(\mathrm{Pt}$ 3), 749-763.

Cover, K. S., Vrenken, H., Geurts, J. J., van Oosten, B. W., Jelles, B., Polman, C. H., Stam, C. J., and van Dijk, B. W. (2006). Multiple sclerosis patients show a highly significant decrease in alpha band interhemispheric synchronization measured using MEG. Neuroimage 29, 783-788.

Damoiseaux, J. S., Rombouts, S. A., Barkhof, F., Scheltens, P., Stam, C. J., Smith, S. M., and Beckmann, C. F. (2006). Consistent resting-state

connectivity underlying clinical symptoms enables the reconciliation of previous localized neuroimaging findings with known anatomical connections of sensorimotor function. The notion of hemispheric competition and the potentially negative influence of the contralesional motor cortex on ipsilesional cortex requires further investigation within this framework. Nonetheless, evaluations of the positive and negative influences acting upon each node of an extended motor network is emerging as an essential technique in the study of motor recovery. Modulation of neural interactions, either through TMS, physical therapy, or pharmacological interventions, may then be directly targeted so that increasingly favorable outcomes may ensue.

\section{ACKNOWLEDGMENTS}

Funded by NIH grants RO1 DC4855, DC6435, DC10145, NS67962, NS64060, and by NSF grant BCS-0926196 (Srikantan S. Nagarajan); American Heart Association (Kelly P. Westlake); and Canadian Institutes of Health Research (postdoctoral fellowship to Kelly P. Westlake).

networks across healthy subjects. Proc. Natl. Acad. Sci. U.S.A. 103 , 13848-13853.

de Haan, W., Pijnenburg, Y. A., Strijers, R. L., van der Made, Y., van der Flier, W. M., Scheltens, P., and Stam, C. J. (2009). Functional neural network analysis in frontotemporal dementia and Alzheimer's disease using EEG and graph theory. BMC Neurosci. 10 101. doi: 10.1186/1471-2202-10-101

DeVetten, G., Coutts, S. B., Hill, M. D. Goyal, M., Eesa, M., O’Brien, B., Demchuk, A. M., and Kirton, A. (2010). Acute corticospinal tract Wallerian degeneration is associated with stroke outcome. Stroke 41 , 751-756.

Dimyan, M. A., and Cohen, L. G. (2010). Contribution of transcranial magnetic stimulation to the understanding of functional recovery mechanisms after stroke. Neurorehabil. Neural Repair 24 , 125-135.

Dong, Y., Dobkin, B. H., Cen, S. Y., Wu, A. D., and Winstein, C. J. (2006). Motor cortex activation during treatment may predict therapeutic gains in paretic hand function after stroke. Stroke 37, 1552-1555.

Ferbert, A., Priori, A., Rothwell, J. C., Day, B. L., Colebatch, J. G., and Marsden, C. D. (1992). Interhemisphericinhibition of the human motor cortex. J. Physiol. $453,525-546$.

Fransson, P. (2005). Spontaneous lowfrequency BOLD signal fluctuations: an fMRI investigation of the restingstate default mode of brain function hypothesis. Hum. Brain Mapp. 26, 15-29.

Friston, K. J., Frith, C. D., Liddle, P. F., and Frackowiak, R. S. (1993). Functional connectivity: the principalcomponent analysis of large (PET) data sets. J. Cereb. Blood Flow Metab. $13,5-14$.

Gerloff, C., Bushara, K., Sailer, A., Wassermann,E.M.,Chen, R., Matsuoka, T., Waldvogel, D., Wittenberg, G. F., Ishii, K., Cohen, L. G., and Hallett, M. (2006). Multimodal imaging of brain reorganization in motor areas of the contralesional hemisphere of well recovered patients after capsular stroke. Brain 129(Pt 3), 791-808.

Gerloff, C., and Hallett, M. (2010). Big news from small world networks after stroke. Brain 133(Pt 4), 952-955.

Geschwind, N. (1965a). Disconnexion syndromes in animals and man. I. Brain 88, 237-294.

Geschwind, N. (1965b). Disconnexion syndromes in animals and man. II Brain 88, 585-544.

Grefkes, C., Nowak, D.A. , Eickhoff, S. B., Dafotakis, M., Kust, J., Karbe, H., and Fink, G. R. (2008). Cortical connectivity after subcortical stroke assessed with functional magnetic resonance imaging. Ann. Neurol. 63, 236-246.

Grefkes, C., Nowak, D. A., Wang, L. E., Dafotakis, M., Eickhoff, S. B., and Fink, G. R. (2010). Modulating cortical connectivity in stroke patients by rTMS assessed with fMRI and dynamic causal modeling. Neuroimage 50, 233-242.

Greicius, M. D., Supekar, K., Menon, V., and Dougherty, R. F. (2009). Restingstate functional connectivity reflects structural connectivity in the default mode network. Cereb. Cortex 19, 72-78.

Guggisberg, A. G ., Honma, S. M., Findlay, A. M., Dalal, S. S., Kirsch, H. E., Berger, M. S., and Nagarajan, S. S (2008). Mapping functional connectivity in patients with brain lesions. Ann Neurol. 63, 193-203. 
Honey, C. J., and Sporns, O. (2008). Dynamical consequences of lesions in cortical networks. Hum. Brain Mapp. 29, 802-809.

Honey, C. J., Sporns, O., Cammoun, L., Gigandet,X., Thiran,J.P., Meuli, R., and Hagmann, P. (2009). Predicting human resting-state functional connectivity from structural connectivity. Proc. Natl. Acad. Sci. U.S.A. 106, 2035-2040.

Hummel, F. C., Steven, B., Hoppe, J., Heise, K., Thomalla, G., Cohen, L. G., and Gerloff, C. (2009). Deficient intracortical inhibition (SICI) during movement preparation after chronic stroke. Neurology 72, 1766-1772.

James, G. A., Lu, Z. L., VanMeter, J. W., Sathian, K., Hu, X. P., and Butler, A. J. (2009). Changes in resting state effective connectivity in the motor network following rehabilitation of upper extremity poststroke paresis. Top. Stroke. Rehabil. 16, 270-281.

Johansen-Berg, H., Rushworth, M. F., Bogdanovic, M. D., Kischka, U., Wimalaratna, S., and Matthews, P. M. (2002). The role of ipsilateral premotor cortex in hand movement after stroke. Proc. Natl. Acad. Sci. U.S.A. 99, 14518-14523.

Kaiser,M., and Hilgetag, C. C. (2004). Edge vulnerability in neural and metabolic networks. Biol. Cybern. 90, 311-317.

Kaiser, M., Hilgetag, C. C., and van Ooyen, A. (2009). A simple rule for axon outgrowth and synaptic competition generates realistic connection lengths and filling fractions. Cereb. Cortex 19, 3001-3010.

Levin, M. F., Kleim, J. A., and Wolf, S. L. (2009). What do motor "recovery" and "compensation" mean in patients following stroke? Neurorehabil. Neural Repair 23, 313-319.

Lotze, M., Markert, J., Sauseng, P., Hoppe, J., Plewnia, C., and Gerloff, C. (2006). The role of multiple contralesional motor areas for complex hand movements after internal capsular lesion. J. Neurosci. 26, 6096-6102.

Lowe, M. J., Phillips, M. D., Lurito, J. T., Mattson, D., Dzemidzic, M., and Mathews, V.P. (2002).Multiple sclerosis: low-frequency temporal blood oxygen level-dependent fluctuations indicate reduced functional connectivity initial results. Radiology 224, 184-192.

Mantini, D., Perrucci, M. G., Del Gratta, C., Romani, G. L., and Corbetta, M. (2007). Electrophysiological signatures of resting state networks in the human brain. Proc. Natl. Acad. Sci. U.S.A. 104, 13170-13175.

Marreiros, A. C., Kiebel, S. J., and Friston, K. J. (2008). Dynamic causal modelling for fMRI: a two-state model. Neuroimage 39, 269-278.
Mechelli, A., Penny, W. D., Price, C. J., Gitelman, D. R., and Friston, K. J. (2002). Effective connectivity and intersubject variability: using a multisubject network to test differences and commonalities. Neuroimage 17, 1459-1469.

Mohammadi, B., Kollewe, K., Samii, A. Krampfl, K., Dengler, R., and Munte, T. F. (2009). Changes of resting state brain networks in amyotrophic lateral sclerosis. Exp. Neurol. 217, 147-153.

Murase, N., Duque, J., Mazzocchio, R., and Cohen, L. G. (2004). Influence of interhemispheric interactions on motor function in chronic stroke. Ann. Neurol. 55, 400-409.

Nakamura, T., Hillary, F. G., and Biswal, B. B. (2009). Resting network plasticity following brain injury. PLoS ONE 4, e8220. doi: 10.1371/journal. pone. 0008220

Nir,Y., Mukamel, R., Dinstein, I., Privman, E., Harel, M., Fisch, L., Gelbard-Sagiv, H., Kipervasser, S., Andelman, F., Neufeld, M. Y., Kramer, U., Arieli, A., Fried, I., and Malach, R. (2008). Interhemispheric correlations of slow spontaneous neuronal fluctuations revealed in human sensory cortex. Nat. Neurosci. 11, 1100-1108.

Nowak, D. A., Grefkes, C., Dafotakis, M., Kust, J., Karbe, H., and Fink, G. R. (2007). Dexterity is impaired at both hands following unilateral subcortical middle cerebral artery stroke. Eur. J. Neurosci. 25, 3173-3184.

Penny, W. D., Stephan, K. E., Mechelli, A., and Friston, K. J. (2004). Modelling functional integration: a comparison of structural equation and dynamic causal models. Neuroimage 23(Suppl. 1), $\mathrm{S} 264-\mathrm{S} 274$

Quaney, B. M., Perera, S., Maletsky, R., Luchies, C. W., and Nudo, R. J. (2005). Impaired grip force modulation in the ipsilesional hand after unilateral middle cerebral artery stroke. Neurorehabil. Neural Repair 19, 338-349.

Reis, J., Swayne, O.B ., Vandermeeren, Y., Camus, M., Dimyan, M. A., HarrisLove, M., Perez, M. A., Ragert, P., Rothwell, J.C., and Cohen, L. G. (2008). Contribution of transcranial magnetic stimulation to the understanding of cortical mechanisms involved in motor control. J. Physiol. 586, 325-351.

Schaechter, J. D., Fricker, Z. P., Perdue, K. L., Helmer, K. G., Vangel, M. G., Greve, D. N., and Makris, N. (2009). Microstructural status of ipsilesional and contralesional corticospinal tract correlates with motor skill in chronic stroke patients. Hum. Brain Mapp. 30, 3461-3474.

Seitz, R. J., Azari, N. P., Knorr, U., Binkofski, F., Herzog, H., and Freund, H. J. (1999). The role of diaschisis in stroke recovery. Stroke 30, 1844-1850.
Serrien, D. J., Strens, L. H., Cassidy, M. J., Thompson, A. J., and Brown, P. (2004). Functional significance of the ipsilateral hemisphere during movement of the affected hand after stroke. Exp. Neurol. 190, 425-432.

Sharma, N., Baron, J. C., and Rowe, J. B. (2009). Motor imagery after stroke: relating outcome to motor network connectivity. Ann. Neurol. 66, 604-616.

Singer, W. (1999). Neuronal synchrony: a versatile code for the definition of relations? Neuron 24, 49-65, 111-125.

Solodkin, A., Hlustik, P., Chen, E. E., and Small, S. L. (2004). Fine modulation in network activation during motor execution and motor imagery. Cereb. Cortex 14, 1246-1255.

Stam, C. J ., de Haan, W., Daffertshofer A., Jones, B. F., Manshanden, I., van Cappellen van Walsum, A. M., Montez, T., Verbunt, J. P., de Munck, J. C., van Dijk, B. W., Berendse, H. W., and Scheltens, P. (2009). Graph theoretical analysis of magnetoencephalographic functional connectivity in Alzheimer's disease. Brain 132(Pt 1), 213-224.

Stinear, C. M., Barber, P. A., Smale, P. R., Coxon, J. P., Fleming, M. K., and Byblow, W. D. (2007). Functional potential in chronic stroke patients depends on corticospinal tract integrity. Brain 130(Pt 1), 170-180.

Stoffers, D., Bosboom, J. L., Deijen, J. B., Wolters, E., Stam, C. J., and Berendse, H.W. (2008). Increased cortico-cortical functional connectivity in early-stage Parkinson's disease: an MEG study. Neuroimage 41, 212-222.

Strens, L. H., Asselman, P., Pogosyan, A., Loukas, C., Thompson, A. J., and Brown, P. (2004). Corticocortical coupling in chronic stroke: its relevance to recovery. Neurology 63, 475-484

van Dellen, E., Douw, L., Baayen, J. C., Heimans, J.J., Ponten, S. C., Vandertop, W. P., Velis, D. N., Stam, C. J., and Reijneveld, J. C. (2009). Long-term effects of temporal lobe epilepsy on local neural networks: a graph theoretical analysis of corticography recordings. PLoS ONE 4, e8081. doi: 10.1371/journal.pone.0008081

van den Heuvel, M. P., Mandl, R. C., Kahn, R. S., and Hulshoff Pol, H. E. (2009). Functionally linked resting-state networks reflect the underlying structural connectivity architecture of the human brain. Hum. Brain Mapp. 30,3127-3141.

Von Monakow, C. (1914). "Die lokalisation im Grosshirn und der Abbau der Funktion durch kortikale Herde Wiesbaden: Bergmann 1914," (Transl. G Harris) in Brain and Behavior I: Mood States and Mind, ed. K. Pribam (Baltimore: Penguin), 27-36.

Walsh, R. R., Small, S. L., Chen, E. E., and Solodkin, A. (2008). Network activa- tion during bimanual movements in humans. Neuroimage 43, 540-553.

Wang, L., Yu, C., Chen, H., Qin, W., He, Y., Fan, F., Zhang, Y., Wang, M., Li, K., Zang, Y., Woodward, T. S., and Zhu, C. (2010). Dynamic functional reorganization of the motor execution network after stroke. Brain 133(Pt 4), 1224-1238.

Ward, N. S., Brown, M. M., Thompson, A. J., and Frackowiak, R. S. (2003). Neural correlates of motor recovery after stroke: a longitudinal fMRI study. Brain 126(Pt 11), 2476-2496.

Ward, N. S., Newton, J. M., Swayne, O. B., Lee, L., Frackowiak, R. S., Thompson, A. J., Greenwood, R. J., and Rothwell, J.C. (2007). The relationship between brain activity and peak grip force is modulated by corticospinal system integrity after subcortical stroke. Eur. J. Neurosci. 25, 1865-1873.

Wernicke, C. (1874). Der aphasische Symptomenkomplex: eine psychologische Studie auf anatomomischer Basis. Breslau: Cohn and Weigert.

Witte, O. W., Bidmon, H. J., Schiene, K., Redecker, C., and Hagemann, G. (2000). Functional differentiation of multiple perilesional zones after focal cerebral ischemia. J. Cereb. Blood Flow Metab. 20, 1149-1165.

Wittenberg, G.F., Bastian,A.J., Dromerick, A. W., Thach, W. T., and Powers, W. J. (2000). Mirror movements complicate interpretation of cerebral activation changes during recovery from subcortical infarction. Neurorehabil. Neural Repair 14, 213-221.

Wu, T., Wang, L., Chen, Y., Zhao, C., Li, K., and Chan, P. (2009). Changes of functional connectivity of the motor network in the resting state in Parkinson's disease. Neurosci. Lett. 460, 6-10.

Conflict of Interest Statement: The authors declare that the research was conducted in the absence of any commercial or financial relationships that could be construed as a potential conflict of interest.

Received: 19 August 2010; accepted: 08 February 2011; published online: 16 March 2011.

Citation: Westlake KP and Nagarajan SS (2011) Functional connectivity in relation to motor performance and recovery after stroke. Front. Syst. Neurosci. 5:8. doi: 10.3389/fnsys.2011.00008

Copyright (c) 2011 Westlake and Nagarajan. This is an open-access article subject to an exclusive license agreement between the authors and Frontiers Media $S A$, which permits unrestricted use, distribution, and reproduction in any medium, provided the original authors and source are credited. 